\title{
Lung ultrasound score predicts outcomes in COVID-19 patients admitted to the emergency department
}

Julio Cesar Garcia de Alencar ${ }^{* *}$ (D), Julio Flavio Meirelles Marchini ${ }^{1}$, Lucas Oliveira Marino', Sabrina Correa da Costa Ribeiro', Cauê Gasparotto Bueno ', Victor Paro da Cunha', Felippe Lazar Neto',

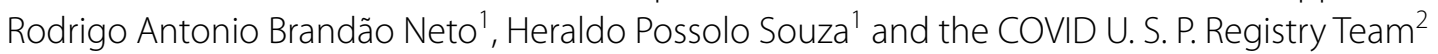

\begin{abstract}
Background: During the COVID-19 pandemic, creating tools to assess disease severity is one of the most important aspects of reducing the burden on emergency departments. Lung ultrasound has a high accuracy for the diagnosis of pulmonary diseases; however, there are few prospective studies demonstrating that lung ultrasound can predict outcomes in COVID-19 patients. We hypothesized that lung ultrasound score (LUS) at hospital admission could predict outcomes of COVID-19 patients. This is a prospective cohort study conducted from 14 March through 6 May 2020 in the emergency department (ED) of an urban, academic, level I trauma center. Patients aged 18 years and older and admitted to the ED with confirmed COVID-19 were considered eligible. Emergency physicians performed lung ultrasounds and calculated LUS, which was tested for correlation with outcomes. This protocol was approved by the local Ethics Committee number 3.990.817 (CAAE: 30417520.0.0000.0068).

Results: The primary endpoint was death from any cause. The secondary endpoints were ICU admission and endotracheal intubation for respiratory failure. Among 180 patients with confirmed COVID-19 who were enrolled (mean age, 60 years; 105 male), the average LUS was 18.7 \pm 6.8 . LUS correlated with findings from chest CT and could predict the estimated extent of parenchymal involvement (mean LUS with $<50 \%$ involvement on chest CT, $15 \pm 6.7$ vs. $21 \pm 6.0$ with $>50 \%$ involvement, $p<0.001$ ), death (AUC 0.72, OR 1.13, 95\% Cl 1.07 to 1.21; $p<0.001$ ), endotracheal intubation (AUC 0.76, OR 1.17,95\% Cl 1.09 to 1.26; $p<0.001$ ), and ICU admission (AUC: 0.71 , OR $1.14,95 \%$ Cl 1.07 to $1.21 ; p<0.001)$.
\end{abstract}

Conclusions: In COVID-19 patients admitted in ED, LUS was a good predictor of death, ICU admission, and endotracheal intubation.

Keywords: COVID-19, Severe acute respiratory syndrome coronavirus 2, Ultrasonography, Critical care, Emergency medicine

*Correspondence: julio.alencar@hc.fm.usp.br

${ }^{1}$ Emergency Department, Hospital das Clínicas da Faculdade de Medicina da Universidade de São Paulo, 255, Dr. Enéas de Carvalho Aguiar st., São Paulo, SP, Brazil

Full list of author information is available at the end of the article

\section{Background}

The novel coronavirus disease 2019 (COVID-19) poses an immense and urgent threat to global health [1]. The entire world is witnessing health care systems, and emergency departments in particular, being overwhelmed by the COVID-19 pandemic [2]. Adequately managing available resources may be the key point to overcoming the surge of patients and saving lives [3]. In this context, tools to assess disease severity and 
prognosis in COVID-19 patients are one of the most important assets in reducing the burden on emergency departments.

Symptoms of COVID-19 vary widely, from asymptomatic disease to severe pneumonia with life-threatening complications [4]. Severe illness usually begins approximately one week after the onset of symptoms, and a striking feature of COVID-19 is the rapid progression to respiratory failure [5]. Patients with severe COVID-19 commonly meet the criteria for acute respiratory distress syndrome (ARDS), which is defined as the acute onset of bilateral infiltrates, severe hypoxemia, and lung edema that is not fully explained by cardiac failure or fluid overload [6]. Even though it can meet the ARDS Berlin definition, COVID-19 pneumonia is a specific disease with peculiar phenotypes, and some investigators propose the presence of two types of patients ("non-ARDS" or type 1, and ARDS or type 2) with different pathophysiologies, distinguishable by chest computed tomography (CT) [7].

Because most patients with severe COVID-19 have pneumonia, imaging is particularly useful for diagnosis and possibly to predict adverse outcomes [8]. Unfortunately, there are downsides. Chest radiography is not sensitive for COVID-19 and usually shows no abnormal findings in the early stages of infection [9]. Chest CT detects early COVID-19 pneumonia with high sensitivity, and small studies have suggested that it can be used to assess disease severity and guide clinical management $[10,11]$. However, obtaining a CT scan requires transporting critically ill patients [12], exposes the patient to radiation [13], and demands that rigorous infection control procedures be followed before scanning subsequent patients [14]. Moreover, CT equipment is not widely available, especially in developing countries [15].

Lung ultrasound is widely used in emergency departments because it is user-friendly, broadly available, lowcost, and has a high accuracy for diagnosing pulmonary diseases [16]. Recent reports suggest that, in COVID-19 patients, lung ultrasound could be useful in several scenarios: to quantify the severity of lung involvement in periodic assessments, to look for findings suggestive of pneumonia, and to monitor the dynamic effects of mechanical ventilation and recruitment maneuvers on lung aeration $[3,17]$.

The lung ultrasound score (LUS) is a semiquantitative score that measures lung aeration loss caused by different pathological conditions $[18,19]$. There are a few prospective studies demonstrating that lung ultrasound can predict outcomes in COVID-19. LUS has been strongly correlated with pulmonary involvement and provides risk stratification, including prediction of need for mechanical ventilation and mortality $[20,21]$.
Within this context, we hypothesized that LUS at hospital admission could predict outcomes in patients with COVID-19.

\section{Methods \\ The aim, design and setting}

This prospective cohort study was conducted from 14 March through 6 May 2020 in the emergency department (ED) of Hospital das Clinicas da Universidade de São Paulo (HC-FMUSP), a 2200-bed urban, academic medical center comprising five institutes and two auxiliary hospitals. During the pandemic, the HC-FMUSP ED has been designated exclusively for the reception and care of patients with COVID-19.

The primary endpoint of the study was death from any cause by 20 July 2020 . The secondary endpoints were any ICU admission and endotracheal intubation for respiratory failure by 20 July 2020 . We chose this date solely to expedite the communication of our findings.

The study protocol was approved by the local Ethics Committee (opinion number 3.990.817; CAAE: 30417520.0.0000.0068), which also waived the need for written informed consent. The present report adheres to the STROBE guidelines.

\section{Patients}

Patients aged 18 years and older who were admitted to the ED with suspected or confirmed COVID-19 were considered eligible. Patients who had advance directives (do not intubate or do not resuscitate) and pregnant women were excluded.

Patients who did not test positive for COVID-19 by reverse-transcriptase polymerase chain reaction (RTPCR) assay of nasopharyngeal swab or tracheal aspiration specimens were also excluded. For the intubation and ICU admission outcomes we excluded patients who were already intubated before we performed lung ultrasound (We still included these patients in mortality analysis).

\section{Research protocol}

After selection, patients were asked for permission to be included in the study. Once permission was granted, a researcher interviewed the patient and collected data through a standardized form using the TeamScope ${ }^{\circledR}$ software (TeamScope Holding Limited, London, England). The following variables were collected: age, sex, day of illness, date of admission, and signs and symptoms on admission.

A second blinded investigator who was not involved in patient care performed the lung ultrasound. Due to the investigators' limited availability, scans were performed only from Monday through Thursday, from 8:00 a.m. to 8:00 p.m. The investigators were aware of the presenting 
symptoms and the most visible physical signs but were blinded to all other clinical information including the radiologic findings.

Subsequently, a third investigator prospectively completed a second questionnaire using the RedCap ${ }^{\circledR}$ software (Vanderbilt University, Nashville, Tennessee, USA) with the following variables collected from electronic medical records: chest CT findings, hospitalization outcome (including hospital discharge and death), need for ICU referral, and need for invasive mechanical ventilation.

Chest CT was performed only for clinical purposes independent of the study protocol. Blinded attending radiologists reported chest CTs as consistent or inconsistent with the most typical pattern described in COVID-19, which includes ground-glass opacities, sometimes with superimposed interlobular septal thickening (crazy paving), consolidations and reversed halo, presenting a bilateral multilobar distribution, predominantly peripheral, with mild predilection for the posterior regions and lower lobes, and gave a visual estimate of the extent of parenchymal involvement (greater or less than 50\%) [10].

\section{LUS protocol}

The investigators were four emergency medicine attending physicians with at least 5 years' experience in pointof-care emergency ultrasonography.

The patient was preferably examined in the sitting position. When this position could not be maintained due to clinical deterioration or poor compliance, the examination was performed in the supine or semirecumbent position. The posterior lung fields were scanned in the sitting position or, when not feasible, by turning the patient onto lateral decubitus position on both sides successively.

We performed lung ultrasound with a Sonosite Edge II portable ultrasound system and a 2- to $5-\mathrm{MHz}$ convex transducer. The examination should start by adjusting the machine to abdominal pre-set to a depth of $15 \mathrm{~cm}$, and the focus should be adjusted to the area of interest. The probe was placed vertically perpendicular to the ribs. Each point was examined for at least one complete respiratory cycle.

The LUS protocol involves the examination of 12 lung regions, performed in around than five minutes in our service: the upper and lower parts of the anterior, lateral, and posterior aspects of the left and right chest wall. Each region is scored according to four ultrasound aeration patterns. For a given region of interest, we allocated points according to the worst ultrasound pattern observed. The final LUS is the sum of points in all 12 regions and ranges from 0 to $36[3,22,23]$. In our study, some terms of the LUS were modified, inspired from Lichtenstein's nomenclature: 0 points-presence of lung sliding with A lines or one or two isolated B lines; 1 point-moderate loss of lung aeration with three or four B lines (septal rockets); 2 points-severe loss of lung aeration with five or more B lines (glass rockets); and 3 points-presence of a hypoechoic poorly defined tissue characterized by complete loss of lung aeration (consolidation) [24].

\section{Statistical analyses}

Data are presented as percentages for categorical variables and the mean \pm standard deviations for continuous variables. All data were tested for normality using the Kolmogorov-Smirnov test. When distribution was normal, we used a two-tailed Student's t-test. We performed logistic regression to explore the associations of LUS with intubation, ICU admission, and mortality. We calculated the area under the receiver operating characteristic curve (AUC) for each regression and accepted statistical significance at $p \leq 0.05$. All analyses were performed using Stata 13 software (College Station, TX, USA).

\section{Results \\ Patients}

During the study period, we admitted 1606 consecutive patients. Of these, 506 patients confirmed COVID-19 by RT-PCR, and 180 were enrolled (Fig. 1). The median age was 60 years, and 105 patients (58\%) were men. The clinical and laboratory characteristics of the patients are summarized in Table 1.

\section{Outcomes}

All patients underwent LUS on the day of emergency department admission. The average LUS was 18.7, with a standard deviation of 6.8 .

We enrolled 180 patients, 109 (60\%) were discharged alive, 61 (33\%) died, and 10 patients (5\%) were still in the hospital at the study endpoint. As of 20 July 2020, 74 patients (56\%) had been treated in the ICU, and 52 (39\%) received invasive mechanical ventilation. Forty-seven patients were already intubated at admission or were intubated shortly after admission, before lung ultrasound could be performed, and were excluded from intubation and ICU analysis. The mean time between lung ultrasound and intubation was $2.1 \pm 1.9$ days with a median of 2 days.

Among the 142 patients who underwent chest $\mathrm{CT}$ on admission, LUS was associated with the extension of COVID-19 pneumonia on CT. The mean LUS in patients with $<50 \%$ involvement on chest $C \mathrm{~T}$ was $15 \pm 6.7$ vs. $21 \pm 6.0$ in those with $>50 \%$ involvement $(p<0.001)$.

Duration of symptoms before admission did not correlate with LUS. We also performed a univariate analysis 


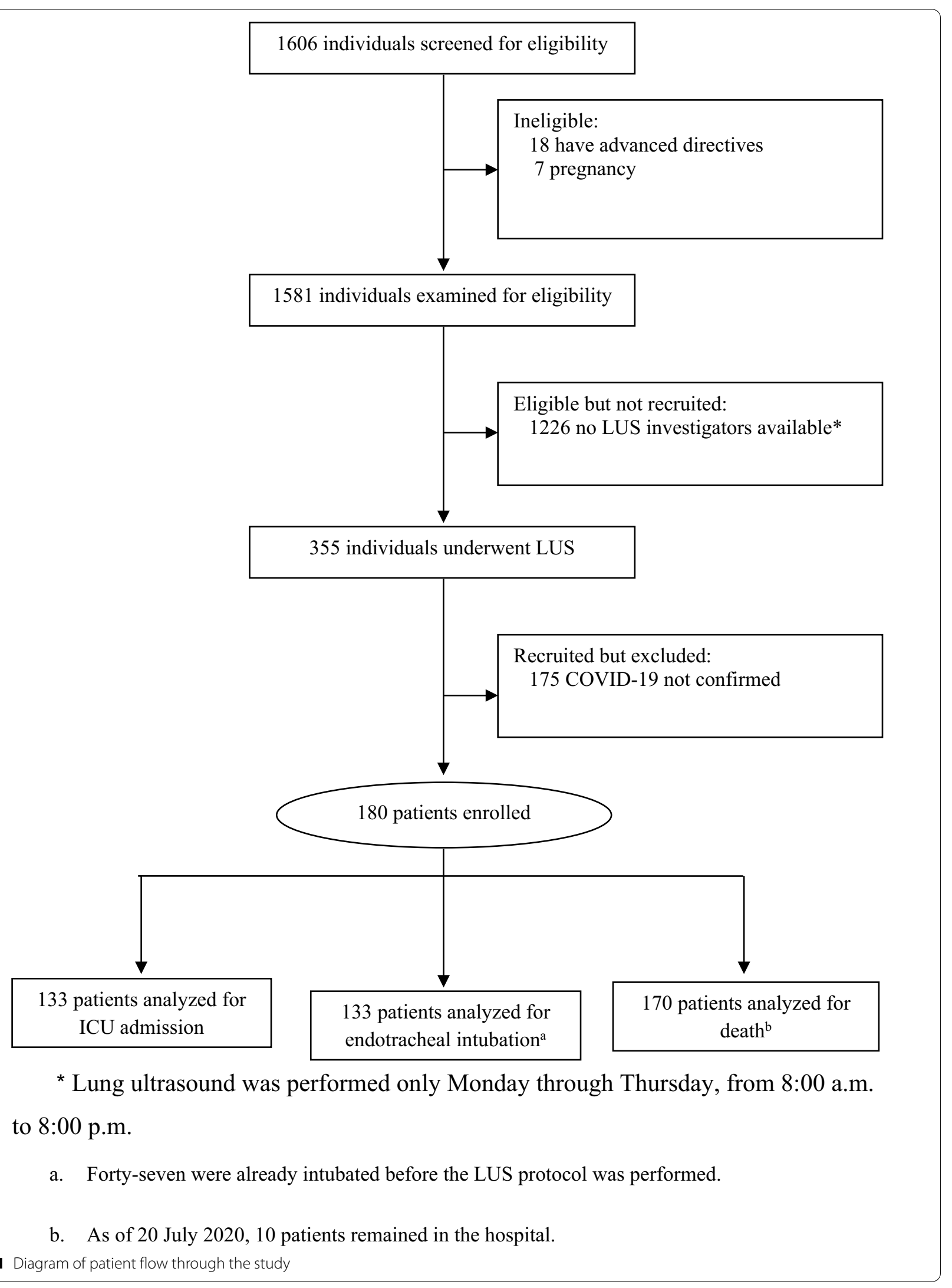


Table 1 Baseline characteristics of patients, vital signs, and laboratory results at admission

\begin{tabular}{|c|c|}
\hline Characteristic $(\mathrm{N}=180)$ & Median (IQR) or N (\%) \\
\hline Age (y) & $60(49-70)$ \\
\hline \multicolumn{2}{|l|}{ Sex } \\
\hline Female & $75(42 \%)$ \\
\hline Male & $105(58 \%)$ \\
\hline \multicolumn{2}{|l|}{ Comorbidities } \\
\hline Hypertension & $98(55 \%)$ \\
\hline Diabetes & $69(39 \%)$ \\
\hline Congestive heart failure & $25(14 \%)$ \\
\hline Chronic respiratory disease & $15(8 \%)$ \\
\hline Chronic and end-stage renal disease & $11(6 \%)$ \\
\hline $\begin{array}{l}\text { Duration of symptoms before hospital admis- } \\
\text { sion (days) }\end{array}$ & $7(5-10)$ \\
\hline Oxygen saturation $<93 \%{ }^{\mathrm{b}}$ & $78(43 \%)$ \\
\hline Oxygen saturation—Median (IQR) & $93(90-96)$ \\
\hline Received supplemental oxygen at triage & $136(76 \%)$ \\
\hline \multicolumn{2}{|l|}{ Type of supplemental oxygen } \\
\hline Nasal cannula & $53(30 \%)$ \\
\hline Nasal cannula oxygen flow & $2.8 \pm 1.4 \mathrm{~L} \mathrm{O}_{2} / \mathrm{min}$ \\
\hline Venturi mask $\left(\mathrm{FiO}_{2}=50 \%\right)$ & $1(1 \%)$ \\
\hline Nonrebreather mask & 35 (19\%) \\
\hline Nonrebreather mask oxygen flow & $11.9 \pm 3.5 \mathrm{~L} \mathrm{O}_{2} / \mathrm{min}$ \\
\hline Mechanical ventilation & $47(27 \%)$ \\
\hline Mechanical ventilation $\mathrm{FiO}_{2}$ & $86.5 \pm 21.8 \%$ \\
\hline Respiratory rate $>24$ breaths $/ \mathrm{min}^{b}$ & $91(51 \%)$ \\
\hline Respiratory rate-median (IQR) & $25(20-30)$ \\
\hline Heart rate $>100$ beats $/ \mathrm{min}$ & $53(29 \%)$ \\
\hline Heart rate-median (IQR) & $92(80-103)$ \\
\hline $\mathrm{PaO}_{2} / \mathrm{FiO}_{2}$ on admission, median (IQR) & $120(64-230)$ \\
\hline LUS $^{\mathrm{a}}$ & $18.7 \pm 6.8$ \\
\hline
\end{tabular}

IQR interquartile range, LUS lung ultrasound score

${ }^{a}$ Mean \pm standard deviation

${ }^{b}$ Most of these patients did not tolerate removal of supplemental oxygen to measure at room air

with symptoms at admission and laboratory tests and found no correlation with mortality in our patients. However, age and bilateral lung involvement $>50 \%$ on chest CT were predictors of death.

As observed in Fig. 2 and Table 2, LUS could predict death, endotracheal intubation, and ICU admission. We plotted AUC to define useful cutoffs for LUS, as shown in Table 3.

\section{Discussion}

The COVID-19 pandemic has brought numerous patients complaining of fever, cough, and dyspnea to ED around the world. Proper assessment of the severity and extent of pulmonary involvement is of paramount importance to select patients who will be admitted to hospital wards or ICUs and thus ensure adequate management of overwhelmed healthcare resources.

In this present study, we analyzed the prognostic value of lung ultrasound in ED COVID-19 patients. We also described a useful tool for lung ultrasound findings that can be summarized in a simple ordinal scoring system (LUS) which was able to discriminate patients' outcomes.

The findings in this study significantly correlate clinical severity of COVID-19 pneumonia and extent of lung pathology detected by LUS, suggesting the utility of LUS in risk stratification of COVID-19 patients and clinical decision-making. The use of LUS to quantify and monitor changes in lung aeration has been described in critically ill patients with ARDS [22]. In COVID-19 patients, contrary to what has been described in ARDS, interstitial patterns and consolidations contribute almost equally to lack of aeration, thus, the severity of respiratory impairment seems to be related to the overall proportion of lung tissue showing ground-glass opacities [7]. Furthermore, the peripheral distribution of lung infiltrates in COVID19 makes lung ultrasound a reliable imaging study [20].

In our study, LUS had a good level of discrimination between admitted patients (including intubation and dead), and increased LUS was associated with worsening disease. LUS predicts mortality with AUC 0.72, and score $\geq 26$ had $90 \%$ specificity for mortality during admission. Interestingly, two recent studies also demonstrated that LUS has a good agreement in the assessment of outcomes in COVID-19 patients: Brahier et al. and Youden et al. showed correlation between LUS and mortality with AUC 0.76 and 0.78 , respectively $[20,21]$.

Lung ultrasound can dynamically assess the ventilation status and provided earlier prediction of pulmonary ventilation status and disease deterioration [22]. In our study, LUS also increased progressively according to clinical severity, and LUS of the intubated was higher than that of the non-intubated group. LUS $\geq 25$ on admission had $90 \%$ specificity for needed intubation, and may be a warning for intubation or exacerbations in critically ill COVID-19 patients in ED.

Moreover, we describe a significant relationship between extent of lung pathology detected by LUS and chest $\mathrm{CT}$. This finding demonstrates that lung ultrasound is a viable instrument, easily performed at the bedside, to evaluate pneumonia severity in COVID-19 patients.

Some limitations of this study must be addressed. First, it was a single-center study conducted at a large academic hospital in São Paulo, Brazil. Second, the level of expertise required to detect small changes in LUS, together with operator dependence, may limit the clinical applicability of lung ultrasound. Third, although lung ultrasound is operator-dependent, we did not test an inter 


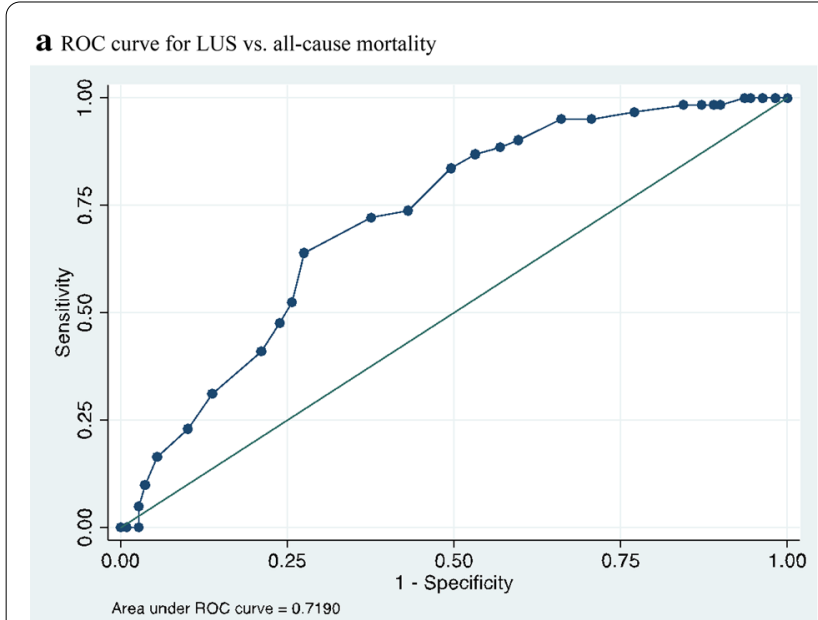

b ROC curve for LUS vs. endotracheal intubation

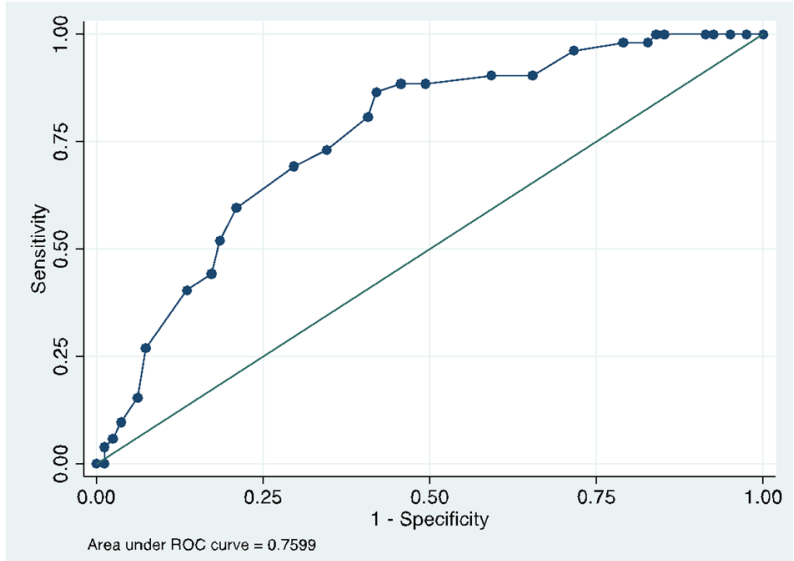

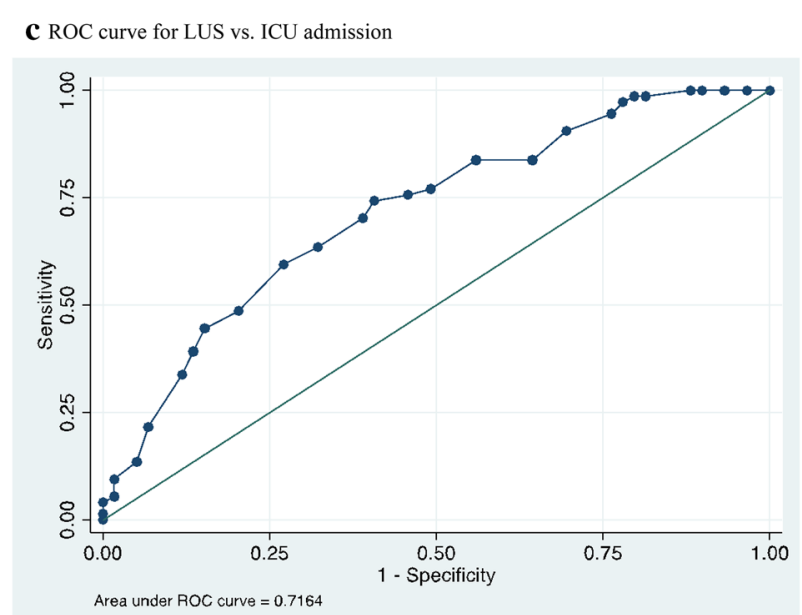

Fig. 2 ROC Curves and Outcomes. a For LUS versus all-cause mortality. b For LUS versus endotracheal intubation. c For LUS versus ICU admission

Table 2 LUS and outcomes in patients with COVID-19

\begin{tabular}{|c|c|c|c|c|}
\hline & Patients & LUS (mean \pm SD) & OR $(95 \% \mathrm{Cl})$ & $p^{* *}$ \\
\hline \multicolumn{5}{|l|}{ Primary outcome } \\
\hline Deceased & $61 / 170(36 \%)$ & $21.6 \pm 4.9$ & $1.13(1.07-1.21)^{*}$ & $<0.001$ \\
\hline Discharged alive & 109/170 (64\%) & $16.7 \pm 4.9$ & & \\
\hline \multicolumn{5}{|c|}{ Secondary outcomes } \\
\hline Intubated & $52 / 133(39 \%)$ & $21.3 \pm 4.9$ & $1.17(1.09-1.26)$ & $<0.001$ \\
\hline Not intubated & $81 / 133$ (61\%) & $15.2 \pm 7.1$ & & \\
\hline Admitted to ICU & $74 / 133(56 \%)$ & $20.0 \pm 5.9$ & $1.14(1.07-1.21)$ & $<0.001$ \\
\hline
\end{tabular}

* Adjustment for age did not change results

** p-values calculated by Student's t-test

intra-observer agreement. To minimize these limitations, experienced physicians performed LUS using a standardized procedure and a pre-defined scoring method. Fourth, the absence of data on patients who remained hospitalized at the date of final data collection may has biased the findings. It would have been better to wait for all patients to achieve a definite outcome; however, because our results are already significant and we believe they are relevant, we chose to sacrifice these data for the sake of reporting our findings more quickly. Lastly, some 
Table 3 LUS and outcome cutoffs

\begin{tabular}{|c|c|c|c|}
\hline & LUS cutoff & Sensitivity & Specificity \\
\hline Discharged alive & $<16$ & $40 \%$ & $90 \%$ \\
\hline Deceased & $\geq 26$ & $23 \%$ & $90 \%$ \\
\hline $\begin{array}{l}\text { Not intubated during hospital } \\
\text { stay }\end{array}$ & $<15$ & $41 \%$ & $90 \%$ \\
\hline Intubated during hospital stay & $\geq 25$ & $27 \%$ & $93 \%$ \\
\hline Not admitted to ICU & $<13$ & $31 \%$ & $91 \%$ \\
\hline Admitted to ICU & $\geq 25$ & $22 \%$ & $93 \%$ \\
\hline
\end{tabular}

authors do not use the LUS and replace it by short but quantitative descriptions of ultrasound disorders [24].

\section{Conclusions}

In this study, despite some limitations, LUS was a good predictor of death, ICU admission, and endotracheal intubation in patients with COVID-19 admitted in ED. This finding can help emergency physicians determine rapidly the patient's disposition. The study provides support for further research, ideally combining clinical, laboratory, and imaging parameters, to estimate the risk of poor outcomes from COVID-19 infection.

\author{
Abbreviations \\ ARDS: Acute respiratory distress syndrome; AUC: Area under the ROC curve; \\ Cl: Confidence interval; COVID 19: Coronavirus disease 2019; CT: Computed \\ tomography; ED: Emergency department; ICU: Intensive care unit; IL-6: \\ Interleukin-6; LUS: Lung ultrasound score; RT-PCR: Reverse-transcriptase \\ polymerase chain reaction.
}

\section{Acknowledgements}

This research was supported by Fundação de Amparo à Pesquisa do Estado de São Paulo (FAPESP).

We thank our colleagues from the Ultrasound Department, who provided insight and expertise that greatly assisted the research.

\section{COVID USP Registry Team}

Fernando Salvetti Valente, MD, Hassan Rahhal, MD, Juliana Batista Rodrigues Pereira, MD, Eduardo Messias Hirano Padrão, MD, Annelise Passos Bispos Wanderley, MD, Bruno Marques, MD, Felipe Liger Moreira, MD, Luz Marina Gomez Gomez, PHD, Millena Gomes Pinheiro Costa, MD, Lucas de Oliveira Utiyama, MD, Felipe Mouzo Bortoleto, MD, Renan Dourado Tinel, MD, Gabriel Martinez, MD, Saionara Maria Nunes Nascimento, MD, Lucas Gonçalves Dias Barreto, MD, Karina Turaça, MD, Debora Lopes Emerenciano, MD, Daniel Rodrigues Ribeiro, MD, Danilo Dias de Francesco, MD, Eduardo Mariani Pires de Campos, MD, Stefany Franhan Barbosa de Souza, MD, Geovane Wiebelling da Silva, MD, Andrew Araujo Tavares, MD, Clara Carvalho de Alves Pereira, MD, Ademar Lima Simões, MD, Gustavo Biz Martins, MD, Leonardo Antonio Coimbra Moreira, MD, Maria Lorraine Silva de Rosa, MD, Pedro Henrique Rodrigues Santana, MD, Thiago Areas Lisboa Netto, MD, Eduardo Padula, MD, Julio Cesar Leite Fortes, MD, Mauricio Ursoline do Nascimento, MD, Rafael Faria Pisciolaro, MD, Rodolfo Affonso Xavier, MD, Marcel Yukio Kamonseki, MD, Patricia Perez Barroso, MD, Rodolfo Avelino de Souza, MD, Yago Henrique Padovan Chio, MD, Edwin Albert D'Souza ${ }^{\top}$, Arthur Petrillo Bellintani ${ }^{\top}$, Rodrigo Cezar Milée ${ }^{\top}$, Rodrigo Werner Toccoli ${ }^{\top}$, Fernanda Máximo Fonseca e Silva ${ }^{\top}$, João Martelleto Baptista ${ }^{\top}$, Marcelo de Oliveira Silva', Giovanna Babikian Costa ${ }^{\top}$, Rafael Berenguer Luna ${ }^{\top}$, Henrique Tibucheski dos Santos', Mariana Mendes Gonçalves Cimatti De Calasans ${ }^{\top}$, Marcelo Petrof Sanches ${ }^{\top}$, Diego Juniti Takamune ${ }^{\top}$, Luiza Boscolo ${ }^{\top}$, Pedro Antonio Araújo Simões ${ }^{\top}$, Manuela Cristina Adsuara Pandolf ${ }^{\top}$, Beatriz Larios Fantinatti ${ }^{\top}$, Gabriel Travessini ${ }^{\top}$, Matheus Finardi Lima de Faria ${ }^{\top}$, Ligia
Trombetta Lima ${ }^{\top}$, Bianca Ruiz Nicolao ${ }^{\top}$, Gabriel de Paula Maroni Escudeiro ${ }^{\top}$, João Pedro Afonso Nascimento', Bruna Tolentino Caldeira', Laura de Góes Campos $^{\top}$, Vitor Macedo Brito Medeiros ${ }^{\top}$, Tales Cabral Monsalvarga ${ }^{\top}$, Isabela Harumi Omori ${ }^{\top}$, Diogo Visconti Guidotte ${ }^{\top}$, Alexandre Lemos Bortolotto ${ }^{\top}$, Rodrigo de Souza Abreu ${ }^{\top}$, Nilo Arthur Bezerra Martins ${ }^{\top}$, and Carlos Eduardo Umehara Juck'.

${ }^{\top}$ Medical student, Faculdade de Medicina da Universidade de São Paulo

\section{Authors' contributions}

JCGA, JFMM, and HPS conceived the study. JCGA, JFMM, SCCR, and RABN performed the lung ultrasound. The COVID USP Registry Team conducted patient interviews and collected data from medical records. JCGA and JFMM ran statistical analyses. JCGA, JFMM, CGB, VPC, FLN, RABN, LOM, and HPS wrote the manuscript. All authors provided critical feedback and helped shape the research, analysis, and manuscript. All authors read and approved the final manuscript.

\section{Funding}

This study was supported by Fundação de Amparo à Pesquisa do Estado de São Paulo (FAPESP), Grants \#2020/04.738-8 and \#2016/14.566-4.

\section{Availability of supporting data}

The data that support the findings of this study are available from the corresponding author JCGA upon reasonable request.

\section{Ethics approval and consent to participate}

The study protocol was approved by the local Ethics Committee (CAPpesq, number 3.990.817; CAAE: 30417520.0.0000.0068), which also waived the need for written informed consent.

\section{Consent for publication}

All authors have read and approved the submission of the manuscript. The manuscript has not been published and is not being considered for publication elsewhere, in whole or in part, in any language.

\section{Competing interests}

The authors declare they have no competing interests.

\section{Author details}

${ }^{1}$ Emergency Department, Hospital das Clínicas da Faculdade de Medicina da Universidade de São Paulo, 255, Dr. Enéas de Carvalho Aguiar st., São Paulo, SP, Brazil. ${ }^{2}$ Medical student, Faculdade de Medicina da Universidade de São Paulo, São Paulo, Brazil.

Received: 18 September 2020 Accepted: 31 December 2020 Published online: 11 January 2021

\section{References}

1. Wynants L, Van Calster B, Bonten MMJ, Collins GS, Debray TPA, De Vos M, et al. Prediction models for diagnosis and prognosis of covid-19 infection: systematic review and critical appraisal. BMJ. 2020;369:m1328.

2. Xie J, Tong Z, Guan X, Du B, Qiu H, Slutsky AS. Critical care crisis and some recommendations during the COVID-19 epidemic in China. Intensive Care Med. 2020;46(5):837-40.

3. Volpicelli G, Lamorte A, Villén T. What's new in lung ultrasound during the COVID-19 pandemic. Intensive Care Med. 2020;46(7):1445-8.

4. Weiss P, Murdoch DR. Clinical course and mortality risk of severe COVID19. The Lancet. 2020;395:1014-5.

5. Berlin DA, Gulick RM, Martinez FJ. Severe Covid-19 [published online ahead of print, 2020 May 15]. N Engl J Med. 2020.

6. ARDS Definition Task Force, Ranieri VM, Rubenfeld GD, Thompson BT, et al. Acute respiratory distress syndrome: the Berlin Definition. JAMA. 2012;307(23):2526-33.

7. Gattinoni L, Chiumello D, Caironi P, et al. COVID-19 pneumonia: different respiratory treatments for different phenotypes? Intensive Care Med. 2020;46:1099-102. 
8. Zu ZY, Jiang MD, Xu PP, Chen W, Ni QQ, Lu GM, et al. Coronavirus disease 2019 (COVID-19): a perspective from China. Radiology. 2020;296(2):E15-25

9. Ng M-Y, Lee EY, Yang J, Yang F, Li X, Wang H, et al. Imaging profile of the COVID-19 infection: radiologic findings and literature review. RadiolCardiothorac Imaging. 2020;2(1):e200034.

10. Bernheim A, Mei $X$, Huang $M$, Yang $Y$, Fayad ZA, et al. Chest $C T$ findings in coronavirus disease-19 (COVID-19): relationship to duration of infection. Radiology. 2020;295:3

11. Colombi D, Bodini FC, Petrini M, Maffi G, Morelli N, Milanese G, et al. Well-aerated lung on admitting chest ct to predict adverse outcome in COVID-19 pneumonia. Radiology. 2020;296(2):E86-96.

12. Beckmann U, Gillies DM, Berenholtz SM, Wu AW, Pronovost P. Incidents relating to the intra-hospital transfer of critically ill patients. Intensive Care Med. 2004;30(8):1579-85.

13. Mayo JR, Aldrich J, Müller NL. Radiation exposure at chest CT: a statement of the Fleischner society. Radiology. 2003;228(1):15-21.

14. Kooraki S, Hosseiny M, Myers L, Gholamrezanezhad A. Coronavirus (COVID-19) outbreak: what the department of radiology should know. J Am CollRadiol. 2020;17(4):447-51.

15. El Khamlichi A. African neurosurgery: current situation, priorities, and needs. Neurosurgery. 2001:48(6):1344-7.

16. Wimalasena Y, Kocierz L, Strong D, et al. Lung ultrasound: a useful tool in the assessment of the dyspnoeic patient in the emergency department. Fact or fiction? Emerg Med J. 2018;35:258-66.

17. Volpicelli G, Elbarbary M, Blaivas M, Lichtenstein DA, Mathis G, Kirkpatrick AW, et al. International evidence-based recommendations for point-ofcare lung ultrasound. Intensive Care Med. 2012;38(4):577-91.
18. Deng $Q$, Zhang $Y$, Wang $H$, Chen L, Yang Z, et al. Semiquantitative lung ultrasound scores in the evaluation and follow-up of critically ill patients with COVID-19: a single-center study. AcadRadiol. 2020;27(10):1363-72.

19. Soummer A, Perbet S, Brisson H, Arbelot C, Constantin JM, Lu Q, et al. Ultrasound assessment of lung aeration loss during a successful weaning trial predicts postextubation distress. Crit Care Med. 2012;40(7):2064-72.

20. Lichter Y, Topilsky Y, Taieb P, et al. Lung ultrasound predicts clinical course and outcomes in COVID-19 patients. Intensive Care Med. 2020:46(10):1873-83.

21. Brahier T, Meuwly J-Y, Pantet O, Vez M-JB, et al. Lung ultrasonography for risk stratification in patients with COVID-19: a prospective observationa cohort study. Clin Infect Dis. 2020. https://doi.org/10.1093/cid/ciaa1408.

22. Bouhemad B, Brisson H, Le-Guen M, Arbelot C, Lu Q, Rouby JJ. Bedside ultrasound assessment of positive end-expiratory pressure-induced lung recruitment. Am J RespirCrit Care Med. 2011;183:341-7.

23. Caltabeloti F, Monsel A, Arbelot C, et al. Early fluid loading in acute respiratory distress syndrome with septic shock deteriorates lung aeration without impairing arterial oxygenation: a lung ultrasound observational study. Crit Care. 2014;18(3):R91.

24. Lichtenstein D. Current misconceptions in lung ultrasound A short guide for experts. Chest. 2019;156(1):21-5.

\section{Publisher's Note}

Springer Nature remains neutral with regard to jurisdictional claims in published maps and institutional affiliations.

\section{Submit your manuscript to a SpringerOpen ${ }^{\circ}$ journal and benefit from:}

- Convenient online submission

- Rigorous peer review

- Open access: articles freely available online

- High visibility within the field

- Retaining the copyright to your article

Submit your next manuscript at springeropen.com 